\title{
Exploring the long-term variability and evolutionary stage of the interacting binary DQ Velorum ${ }^{\star}$
}

\author{
D. Barría ${ }^{1}$, R. E. Mennickent ${ }^{1}$, D. Graczyk ${ }^{1}$, and Z. Kołaczkowski ${ }^{2}$
}

\author{
1 Universidad de Concepción, Departamento de Astronomía, Casilla 160, Concepción, Chile \\ e-mail: [dbarria; rmennick; darek]@astro-udec.cl \\ 2 Instytut Astronomiczny Uniwersytetu Wroclawskiego, 51-622 Wrocław, Poland \\ e-mail: kolaczk@astro1.astro.uni.wroc.pl
}

Received 10 September 2013 / Accepted 26 May 2014

\begin{abstract}
Aims. In order to better understand the double periodic variable (DPV) phenomenon, we analyse a series of optical spectra of the DPV system DQ Velorum during much of its long-term cycle. In addition, we investigate the evolutionary history of DQ Vel using theoretical evolutionary models to obtain the best representation for the current observed stellar and orbital parameters of the binary. We investigate the evolution of DQ Vel through theoretical evolutionary models to estimate the age and the mass transfer rate which are then compared with those of its twin V393 Scorpii.

Methods. We subtracted the donor star contribution from the composite spectra of DQ Vel using a synthetic spectrum as a donor template. Donor-subtracted spectra covering around $60 \%$ of the long-term cycle allowed us to investigate time-modulated spectral variations of the gainer star plus the disc. We used Gaussian fits to measure the equivalent widths (EWs) of Balmer and helium lines in the separated spectra during the long-term cycle and thus analyse EW variabilities. We compared the observed stellar parameters of the system with a grid of theoretical evolutionary tracks computed under a conservative and a non-conservative evolution regime. Results. We have found that the EW of Balmer and helium lines in the donor-subtracted spectra are modulated with the long-term cycle. We observe a strengthening in the EWs in all analysed spectral features at the minimum of the long-term cycle which might be related to an extra line emission during the maximum of the long-term variability. Difference spectra obtained at the secondary eclipse support this scenario. We have found that a non-conservative evolutionary model where DQ Vel has lost mass at some stage of its binary history, is a better representation of the current observed properties of the system. The best evolutionary model suggests that DQ Vel has an age of $7.40 \times 10^{7} \mathrm{yr}$ and is currently in a low mass transfer rate $\left(-9.8 \times 10^{-9} M_{\odot} / \mathrm{yr}\right)$ stage, after a mass transfer burst episode. Comparing the evolutionary stages of DQ Vel and V393 Sco we observed that the former is an older system with a lower mass transfer rate. This might explain the differences observed in the physical parameters of their accretion discs.
\end{abstract}

Key words. binaries: eclipsing - stars: evolution - stars: early-type

\section{Introduction}

DQ Velorum is an eclipsing binary $(V \sim 10.7)$ with an orbital period of $6.08 \mathrm{~d}$; it belongs to the group of double periodic variables (DPVs). This group of interacting binaries was discovered after a photometric search for Be stars in the OGLE-II database. They are characterised by a defined relation between the orbital period $\left(P_{\mathrm{o}}\right)$ and a second observed long-term periodicity $\left(P_{1}\right)$ such that $P_{1}=\eta \times P_{\mathrm{o}}$ (Mennickent et al. 2003; Mennickent \& Kołaczkowski 2010; Poleski et al. 2010). At present, more than 200 DPVs have been discovered in the Milky Way and the Magellanic Clouds. The orbital and long-term periods calculated for the 13 Galactic DPVs known so far, allowed us to find $\eta=32.7$ for the Milky Way DPVs (Mennickent et al. 2012a). In the current scenario, the long-term periodicity is interpreted as cycles of mass loss from the binary into the interstellar medium. After a deep spectroscopic analysis of the DPV system V393 Sco, Mennickent et al. (2012b) find that the long-term variability could be explained in terms of an anisotropic wind of variable strength whose emisivity is modulated with the long

* The reduced spectra are only available at the CDS via anonymous ftp to cdsarc.u-strasbg. fr (130.79.128.5) or via

http://cdsarc.u-strasbg.fr/viz-bin/qcat?]/A+A/567/A140 cycle. However, the mechanism(s) involved in the wind strength variability are still unclear.

Using ASAS $^{1}$ photometric data of DQ Vel, Michalska et al. (2010) detected an additional long photometric variability of $188.9 \mathrm{~d}$ which agrees with the previously mentioned $P_{1} / P_{\mathrm{o}} \mathrm{DPV}$ relation. Using the data obtained after a photometric and spectroscopic campaign carried out during 2008-2011, Barría et al. (2013) determined the fundamental stellar and orbital parameters of this binary. Their techniques involved the separation of the DQ Vel composite spectra, radial velocity (RV) measurements, and a multicomponent fit to the $V$-band ASAS light curve. The authors argued that DQ Vel is a semidetached system consisting of a more massive B3V primary star (hereafter gainer) and an A1III secondary star (hereafter donor) plus an extended accretion disc around the gainer. They obtained a spectroscopic mass ratio $q=0.31 \pm 0.03$, masses of $M_{\mathrm{d}}=2.2 \pm$ $0.2 M_{\odot}$ and $M_{\mathrm{g}}=7.3 \pm 0.3 M_{\odot}$, radii $R_{\mathrm{d}}=8.4 \pm 0.2 R_{\odot}$ and $R_{\mathrm{g}}=3.6 \pm 0.2 R_{\odot}$, and temperatures of $T_{\mathrm{d}}=9400 \pm 100 \mathrm{~K}$ and $T_{\mathrm{g}}=18500 \pm 500 \mathrm{~K}$ for the stellar components. In addition, the physical and geometric parameters for the accretion disc were obtained. Interestingly, strong similarities were found

\footnotetext{
http://www.astrouw.edu.pl/asas/. For further details read Pojmanski (1997).
} 
Table 1. Details of the new spectra included in this study.

\begin{tabular}{lcccccccc}
\hline \hline Observatory & Telescope/Instrument & UT date & HJD-2450000 & $\phi_{\mathrm{o}}$ & $\phi_{\mathrm{l}}$ & Exp time(s) & $\begin{array}{c}S / N \\
\begin{array}{c}\text { Wavelength } \\
\text { coverage }(\AA)\end{array}\end{array}$ \\
& & & & & & & & \\
\hline Las Campanas & Du Pont/echelle & 2012-Jun.-24 & 6103.473 & 0.16 & 0.13 & 120 & 65 & $3630-5260$ \\
Las Campanas & Du Pont/echelle & 2012-Jun.-25 & 6104.448 & 0.32 & 0.14 & 120 & 77 & $3630-5260$ \\
Las Campanas & Du Pont/echelle & 2012-Jun.-26 & 6105.461 & 0.48 & 0.14 & 120 & 65 & $3630-5260$ \\
La Silla & MPG/FEROS & 2012-May-10 & 6057.662 & 0.63 & 0.89 & 600 & 50 & $3530-9220$ \\
La Silla & MPG/FEROS & 2013-Feb.-19 & 6342.652 & 0.48 & 0.40 & 1500 & 55 & $3530-8850$ \\
Las Campanas & Clay/MIKE & 2011-Apr.-05 & 5657.483 & 0.84 & 0.77 & 600 & 47 & $3450-5050(\mathrm{~b})$ \\
& & & & & & & & $4850-9150(\mathrm{r})$ \\
\hline
\end{tabular}

Notes. $\phi_{\mathrm{o}}$ is the orbital phase and $\phi_{1}$ corresponds to the long cycle phase. The $\mathrm{S} / \mathrm{N}$ ratio was measured at the continuum level using the ranges

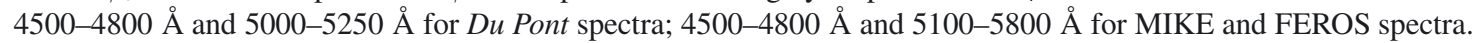

in the stellar parameters of DQ Vel and the well-studied DPV system V393 Sco (Mennickent et al. 2010; 2012a,b). However, the two systems show different geometric and physical properties in their accretion discs, which might be related to different evolutionary stages.

The growing collection of discovered DPVs suggests that they represent a significant stage in the lifetime of a binary. Around $30 \%$ of them are eclipsing and only $2 \%$ have been studied spectroscopically (V393 Sco, DQ Vel, OGLE05155332-6925581, AU Mon (Barria, in prep.), and HD 170582 (Mennickent, in prep.)). In this sense, it becomes important to extend the number of well studied systems to investigate the nature and evolution of DPVs. To do this, main physical properties of the binary components together with spectroscopic analysis during the long-term cycle are required.

In this contribution we present the first spectroscopic analysis of DQ Vel during much of its long photometric cycle. Our analysis includes composite and separated spectra in different orbital and long phases. We also investigate the evolutionary history of DQ Vel and compare these results with evolutionary studies of V393 Sco.

\section{Data analysis}

In this analysis we use the sample of 46 optical spectra previously studied by Barría et al. (2013), supplemented by six additional spectra collected during recent years. A detailed description and reduction processes for the first group of spectra are given in Table 1 and Sect. 2 of the aforementioned paper. The six additional optical spectra were obtained at different instruments/telescopes: three were collected during three nights in June 2012 with the echelle spectrograph at the $2.5 \mathrm{~m}$ Irénée $\mathrm{du}$ Pont telescope located at the Las Campanas Observatory, two spectra were obtained with the echelle spectrograph FEROS mounted at the $2.2 \mathrm{~m} \mathrm{ESO/MPG}$ telescope at La Silla, and one spectrum was collected with the echelle spectrograph MIKE mounted at the $6.5 \mathrm{~m}$ Clay telescope in Las Campanas Observatory. A summary of these spectra is given in Table 1. The orbital and long cycle phases were calculated according to the following ephemerides (Barría et al. 2013):

$$
\begin{aligned}
T_{\text {min,orb }} & =\text { HJD } 2453407.60(2)+6.083299(7) \times E \\
T_{\text {max }, \text { long }} & =\text { HJD } 2453437.20(16)+188.7(2) \times E .
\end{aligned}
$$

In Fig. 1 we show the histograms for the orbital and long cycle phases covered by the total spectra analysed in this paper. Our observations cover $90 \%$ of the orbital variability and $60 \%$ of the long-term periodicity. The Du Pont/echelle and the MPG/FEROS spectra listed in Table 1 were subjected to the same reduction processes described in Barría et al. (2013). The spectral range for the $D u$ Pont/echelle spectra is 3630-5260 $\AA$. The MPG/FEROS spectra has a wavelength coverage from $3530 \AA$ to around $9000 \AA$.

The MIKE instrument is a double echelle spectrograph comprised of two arms (red and blue) with independent CCDs. The standard wavelength coverage for the blue arm is around 3350-5000 $\AA$ and 4900-9500 $\AA$ for the red arm. The instrument resolutions (for one arc-second slit) are 28000 and 22000 for the blue and red sides, respectively. An internal thorium argon lamp is used for wavelength calibration. The standard reduction processes like flat and bias correction, wavelength calibration, continuum normalisation, order merging, and cosmic rays subtraction were applied using IRAF tools ${ }^{2}$. The reduction processes were applied separately in the blue and red sides of the MIKE spectrum. Heliocentric corrections were applied to all spectra listed in Table 1 .

\section{Results and discussion}

\subsection{Long cycle spectroscopic analysis}

Donor and gainer RVs were measured for the spectra listed in Table 1. To compute the donor RVs, we used a donor template spectrum and apply a cross correlation process (CCR) using the IRAF fxcor task in the 4350-5000 A wavelength range where we find more lines coming from the donor. We selected from a grid of synthetic spectra, a donor template spectrum with $T_{\mathrm{d}}=9400 \mathrm{~K}, \log g_{\mathrm{d}}=2.9, v_{\mathrm{r}} \sin i=75 \mathrm{~km} \mathrm{~s}^{-1}$, and solar metallicity, according to the donor parameters found by Barría et al. (2013). The grid of synthetic spectra was computed using atmospheric models with the line-blanketed LTE ATLAS9 code (Kurucz 1993), which treats line opacity with opacity distribution functions (ODFs). The Kurucz models are constructed assuming plane-parallel geometry and hydrostatic and radiative equilibrium of the gas. The synthetic spectra were computed with the SYNTHE code (Kurucz 1993). Both codes, ATLAS9 and SYNTHE, were ported under GNU Linux by Sbordone (2005) and are available online ${ }^{3}$. The atomic data were taken from Castelli \& Hubrig $(2004)^{4}$. The theoretical models were obtained for effective temperatures from $6000 \mathrm{~K}$ to $19000 \mathrm{~K}$ with steps of $100 \mathrm{~K}$ and for surface gravities from $2.0 \mathrm{dex}$ to

2 IRAF (http://iraf.noao.edu/) is distributed by the National Optical Astronomy Observatories and operated by the Association of Universities for Research in Astronomy Inc., under cooperative agreement with the National Science Foundation.

3 http://www.user.oat.ts.astro.it/atmos/

4 http://www.user.oat.ts.astro.it/castelli/grids.html 
D. Barría et al.: Exploring the long-term variability and the evolutionary stage of DQ Velorum
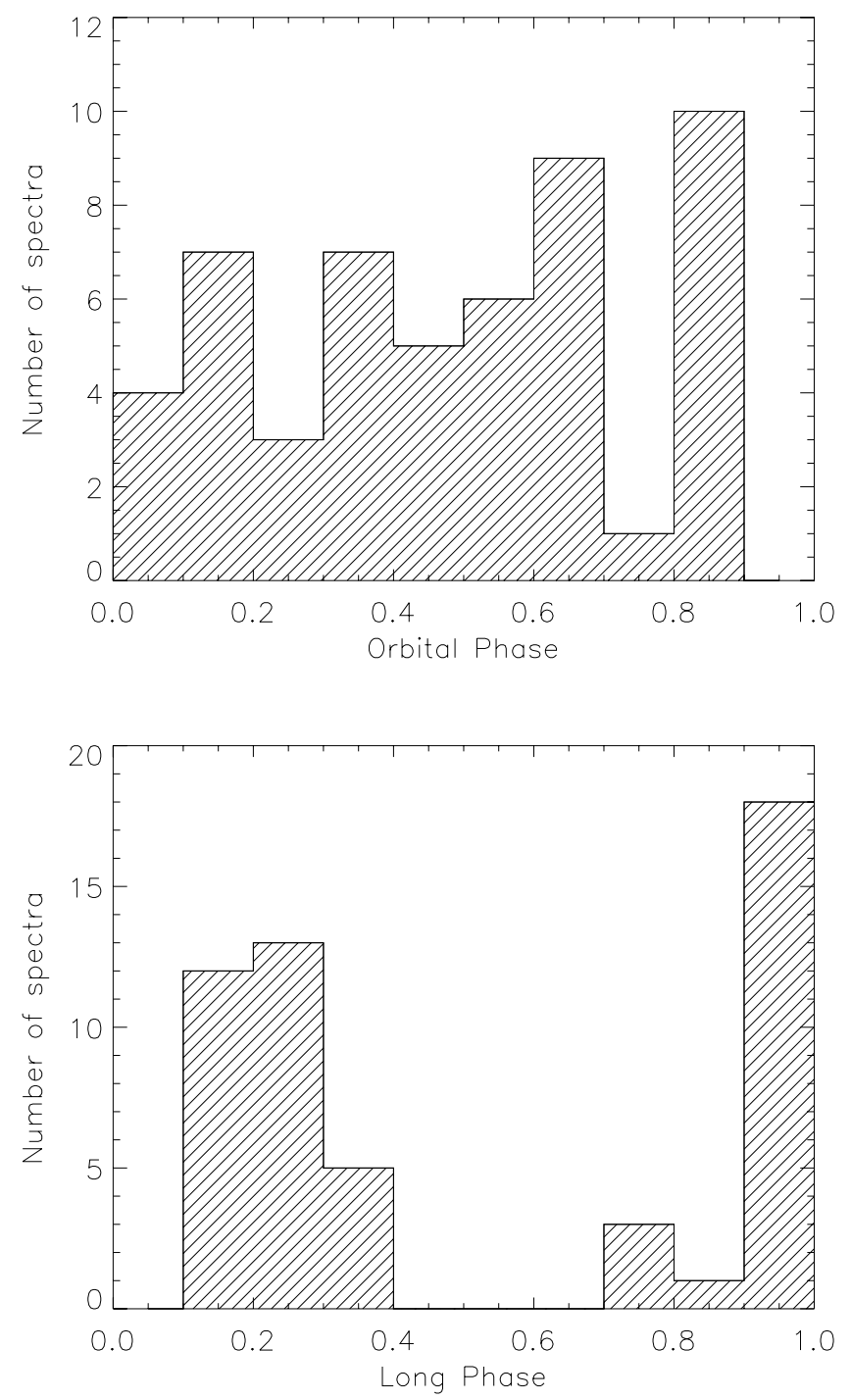

Fig. 1. Top panel: number of spectra per bin of 0.1 orbital phases. Bottom panel: number of spectra per bin of 0.1 long phases.

4.5 dex with steps of 0.1 dex. Solar metallicities and those 0.5 dex higher were taken into account. The grid of synthetic spectra was calculated for different rotation velocities, $v_{\mathrm{r}} \sin i=0$, $25,50,75,100$, and $120 \mathrm{~km} \mathrm{~s}^{-1}$.

We observe that the He I absorption lines move in anti-phase with the donor RVs, which agrees with our previous work. This suggests that these lines originated in or close to the gainer star. Because of the short wavelength coverage of the $\mathrm{Du}$ Pont/echelle spectra (3630-5260 ̊), the gainer RVs are calculated using Gaussian fits to the He I 4026.191/4387.929/4471.479 $\AA$ lines and then computing their Doppler shifts. The gainer RVs for the individual spectra are obtained taking the mean value of the velocities calculated for each of the He I lines. Final RVs for the donor $\left(\mathrm{RV}_{\mathrm{d}}\right)$ and gainer $\left(\mathrm{RV}_{\mathrm{g}}\right)$ are listed in Table 2 .

Remarkable variabilities in the spectra of DQ Vel are observed along the orbital and long-term phases. To analyse the long-term behaviour of the gainer star and the disc, we subtract from each spectra the donor template spectrum multiplied by a light contribution factor obtained from the light curve model. These factors depend on the orbital phase and are calculated for a specific wavelength range around a spectral line (for further details see Barría et al. 2013, Sect. 3.2).
Table 2. Calculated RVs for the spectra listed in Table 1.

\begin{tabular}{lccc}
\hline \hline HJD-2 450 000 & $\phi_{\mathrm{o}}$ & $\mathrm{RV}_{\mathrm{d}}\left(\mathrm{km} \mathrm{s}^{-1}\right)$ & $\mathrm{RV}_{\mathrm{g}}\left(\mathrm{km} \mathrm{s}^{-1}\right)$ \\
\hline 6103.473 & 0.160 & $98.17 \pm 0.88$ & $-77.86 \pm 14.92$ \\
6104.448 & 0.320 & $131.09 \pm 2.80$ & $-47.75 \pm 8.45$ \\
6105.461 & 0.486 & $18.34 \pm 0.06$ & $-79.11 \pm 5.20$ \\
6057.662 & 0.630 & $-121.63 \pm 0.93$ & $9.63 \pm 3.16$ \\
6342.652 & 0.480 & $5.27 \pm 0.11$ & $-24.24 \pm 10.58$ \\
5657.483 & 0.846 & $-137.97 \pm 1.55$ & $34.92 \pm 7.41$ \\
\hline
\end{tabular}

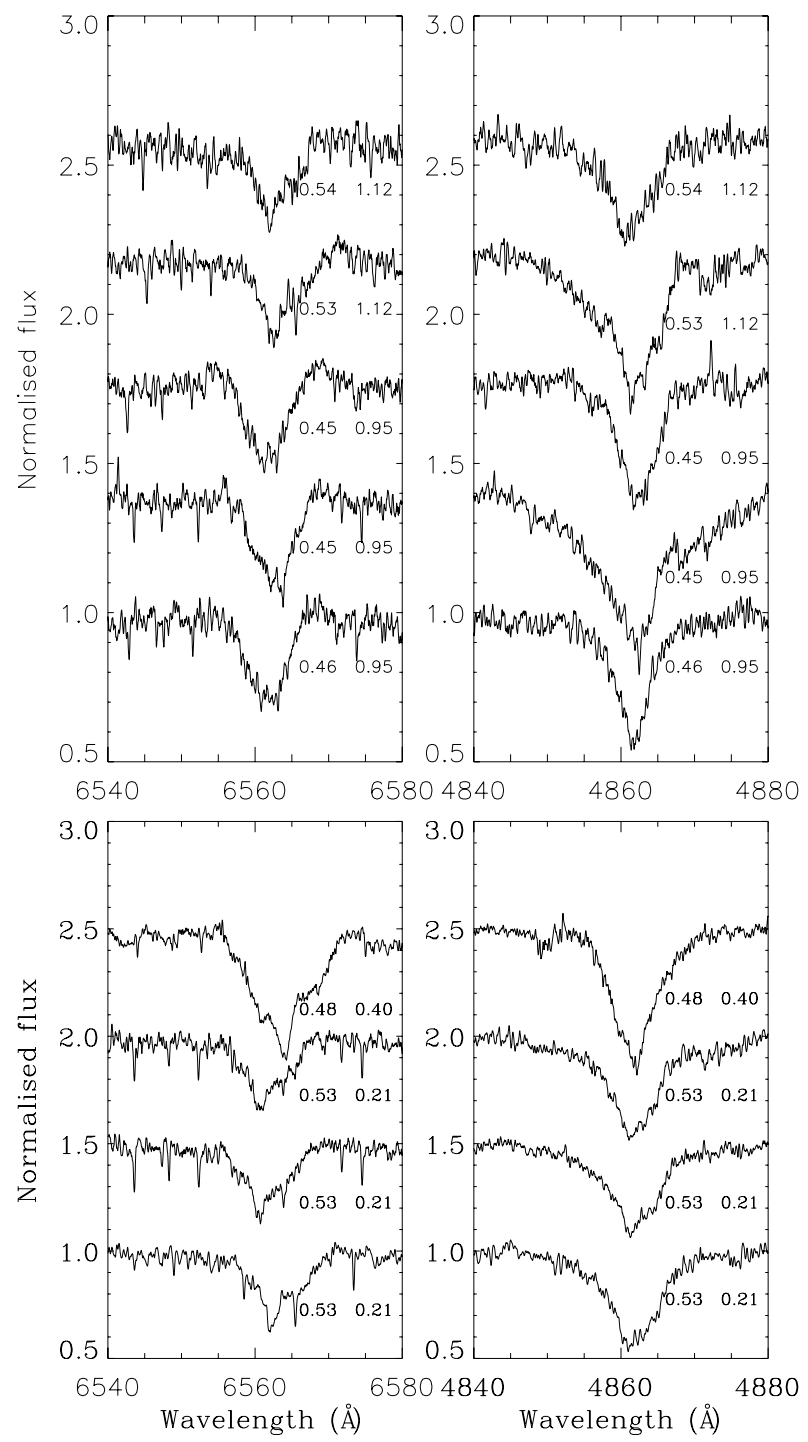

Fig. 2. Donor-subtracted spectra of DQ Vel in the $\mathrm{H} \alpha$ (left panels) and $\mathrm{H} \beta$ (right panels) regions around the secondary eclipse. Spectra are shown at the maximum of the long-term cycle (upper panels) and on the way to the minimum of the long-term variability (lower panels). Labels indicate orbital (left) and long cycle (right) phases. The spectra were offset for clarity.

In Figs. 2 and 3 we show selected donor-subtracted spectra in the $\mathrm{H} \alpha, \mathrm{H} \beta, \mathrm{He} \mathrm{I} 5875, \AA$ and He I $6678 \AA$ regions around the mid-eclipse $\phi_{\mathrm{o}} \sim 0.5$. At the upper panels, spectra along the maximum of the long-term cycle are shown. Here, weak doublepeaked emission of variable strength are seen in the $\mathrm{H} \alpha$ profiles. These emissions can reach velocities up to $\pm 350 \mathrm{~km} \mathrm{~s}^{-1}$. Just after the maximum of the long-term variability $\left(\phi_{1} \sim 1.1\right)$, we observe a weakness of the central absorption in all profiles. The 


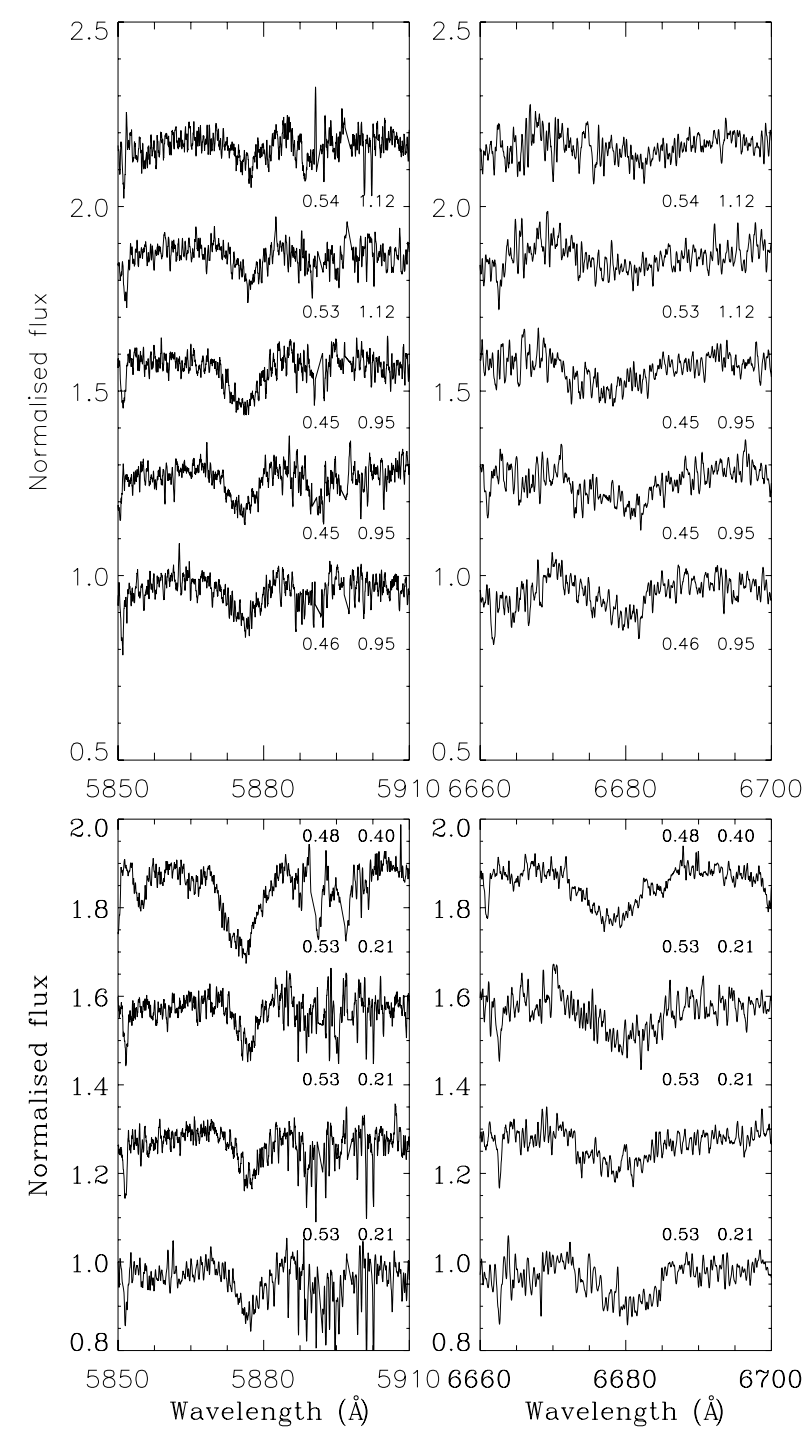

Fig. 3. Donor-subtracted spectra of DQ Vel in the He I 5875 (left panel) and HeI6678 (right panel) regions around the secondary eclipse. Labels indicate orbital (left) and long cycle (right) phases.

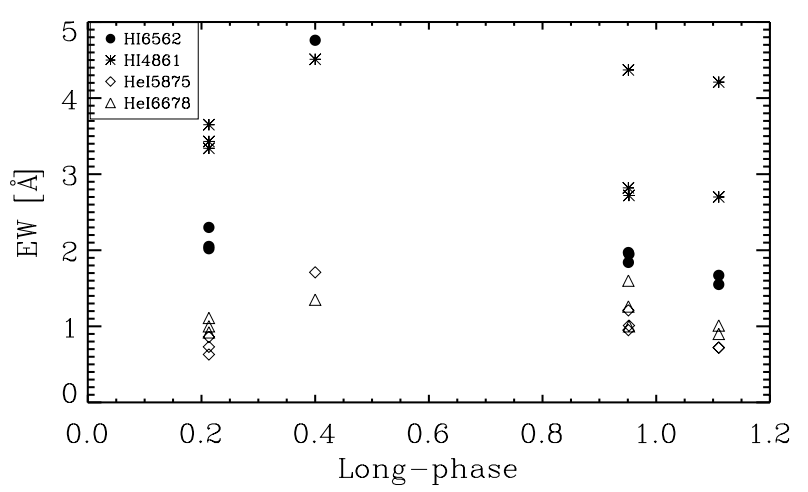

Fig. 4. Equivalent width of the $\mathrm{H} \alpha, \mathrm{H} \beta, \mathrm{He} \mathrm{I} 5875 \AA$ and $\mathrm{He} \mathrm{I} 6678 \AA$ lines along the long-term cycle and around the secondary eclipse.

case of the He I $6678 \AA$ line is remarkable; it seems to dissapear at this long-term phase, as can be seen at the upper-right panel in Fig. 3. Asymmetric $\mathrm{H} \alpha$ and $\mathrm{H} \beta$ profiles are observed at different long-term phases (see e.g. the spectra at $\phi_{1}=0.95,1.12$ in Fig. 3). The origin of these features is unknown but might be related to an asymmetric distribution of the circumstellar matter at different epochs.

In the lower panels of Figs. 2 and 3, we show spectra toward the minimum of the long-term variability $\left(0.2<\phi_{1}<0.4\right)$. We observe a strengthening of the central absorption at $\phi_{1}=0.4$ in all profiles. We measure the equivalenth width (EW) of the central absorption for the spectral lines displayed in Figs. 2 and 3 along the long-term cycle. On average, higher EW values are found during the minimum of the long-term cycle as can be seen in Fig. 4. To check if this pattern is a general trend, we measured the EWs of $\mathrm{H} \alpha, \mathrm{H} \beta$, He I $5875 \AA$, and He I $6678 \AA$ lines for all donor-subtracted spectra during the maximum and the minimum of the long-term variability. As can be seen in Fig. 5, during the minimum of the long-term we observe an increase in the EWs, more significant at the main eclipse $\left(\phi_{\mathrm{o}} \sim 0.0\right)$ when the gainer and the disc are hidden by the donor star. A similar pattern is also detected in the $\mathrm{H} \gamma$ and $\mathrm{H} \delta$ profiles, as well as in other helium lines. An extra global emission such as that suggested by Mennickent et al. (2012a) during the high state of the long cycle could explain this variability pattern.

\subsection{The OI 7773 line and DACs}

Donor-subtracted spectra showing the time variability of the O I $7773 \AA$ line during the orbital and long cycle are displayed in Fig. 6. Radial velocity measurements of the central absorption of OI7773 $\AA$ in the composite spectra show that it is in phase with the donor star. The half-amplitude of the RV curve is $136.1 \pm 3.8 \mathrm{~km} \mathrm{~s}^{-1}$ for a circular-orbit solution. This measure is around $25 \%$ lower than the mean half-amplitude value for the donor RV curve found in our previous paper. This can be explained considering that the O I $7773 \AA$ RV curve was obtained using only five RV measurements from the spectra available at this wavelength range. Moreover, the O I $7773 \AA$ absorption line is broader, asymmetric, and affected by enhanced blue/red absorption wings.

Following the tendency of Balmer and He lines, we observe a deeper and broader central absorption of the O I $7773 \AA$ line at $\phi_{1}=0.4$ and a weakness of this spectral feature at $\phi_{1}=0.88$. Blue depression wings are observed in three spectra at orbital phases $\phi_{0}=0.32,0.48$ and 0.65 and at different long-term phases. Here, weak discrete absorption components (DACs) at the blue $\left(\phi_{\mathrm{o}}=0.48,0.65\right)$ and at the red side $\left(\phi_{\mathrm{o}}=0.32\right)$ of the line have been detected. Discrete absorption components are observed as peculiar line profiles in hot $\mathrm{OB}, \mathrm{Oe}$, and Be-type stars. These components have been interpreted as absorption lines created at different density regions moving radially at distinct velocities (Danezis et al. 2003). Mennickent et al. (2012b) have interpreted DACs observed in the DPV system V393 Sco as signatures of a clumpy wind emerging from the disc in the direction of the binary motion. Interestingly, no DACs are observed at $\phi_{\mathrm{o}}=0.84\left(\phi_{1}=0.76\right)$ and neither blue or red enhanced absorption wings. Although additional spectra are required to confirm this pattern, it seems that enhanced absorption wings and DACs are correlated. A possible explanation for this might be related to the hot spot/line, which reaches its maximum visibility around $\phi_{\mathrm{o}}=0.84$. If the DACs and the enhanced absorption wings are related to a wind, then the hot spot may act veiling the wind effects, especially if the strength of the wind is weak. Considering that DACs are observed only at three of the five spectra available at this wavelength range, these results are similar to those observed by Mennickent et al. (2012b) in spectroscopic studies of V393 Sco. 
D. Barría et al.: Exploring the long-term variability and the evolutionary stage of DQ Velorum
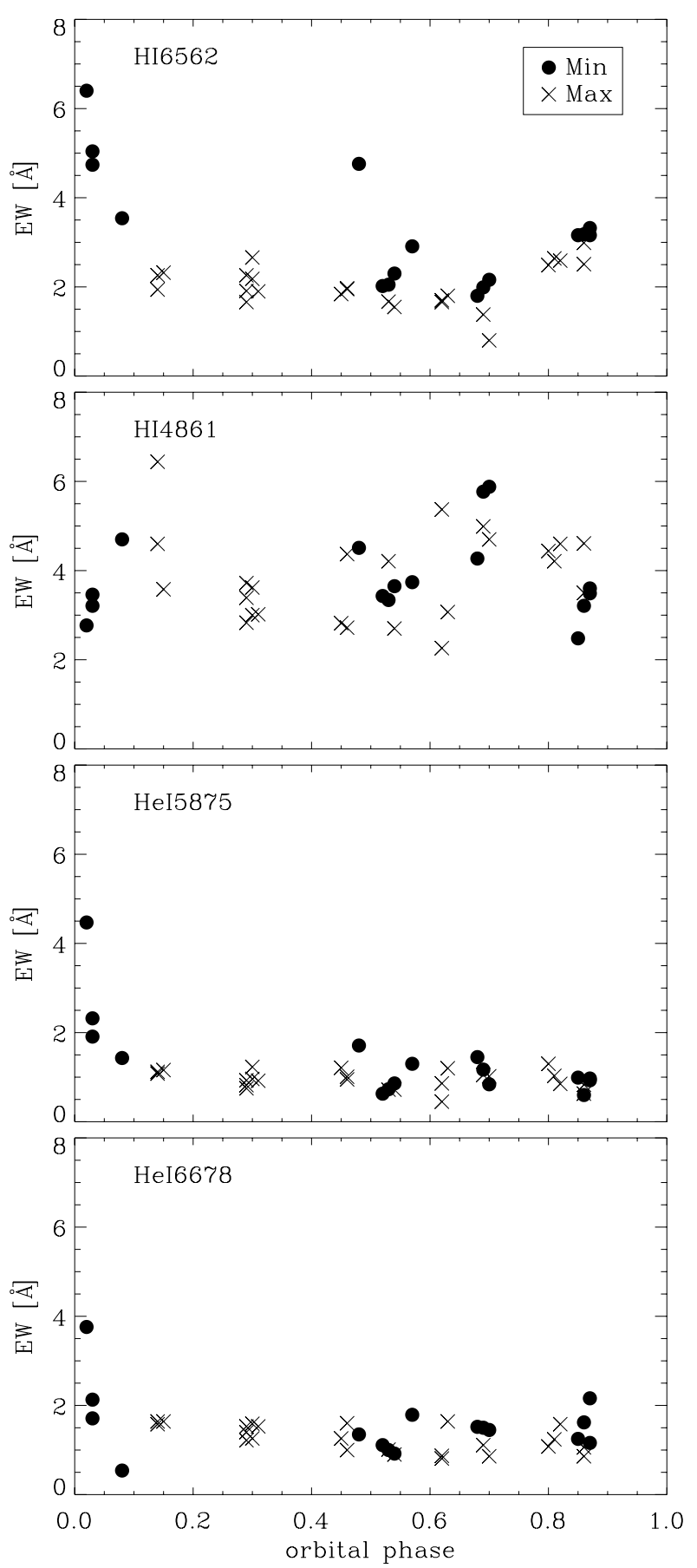

Fig. 5. Equivalent width of the $\mathrm{H} \alpha, \mathrm{H} \beta, \mathrm{He} \mathrm{I} 5875 \AA$ and He I $6678 \AA$ lines along the orbital phase at the maximum and minimum of the long cycle.

\subsection{Difference profiles}

Donor-subtracted spectra of DQ Vel along the orbital motion show that the intensity of the $\mathrm{H} \alpha$ central absorption is weakened outside the eclipses (see Fig. 6, Barría et al. 2013). This can be related to the extra-emission component coming from the interaction region between the gas stream and the accretion disc. To minimise this emission contribution and thus investigate other possible extra absorption/emission features, we analyse a series of higher $\mathrm{S} / \mathrm{N}$ spectra around the secondary eclipse. A sample of $\mathrm{H} \alpha$ composite spectra of DQ Vel at the mid-eclipse are displayed in Fig. 7. Here, weak emission components in the wings

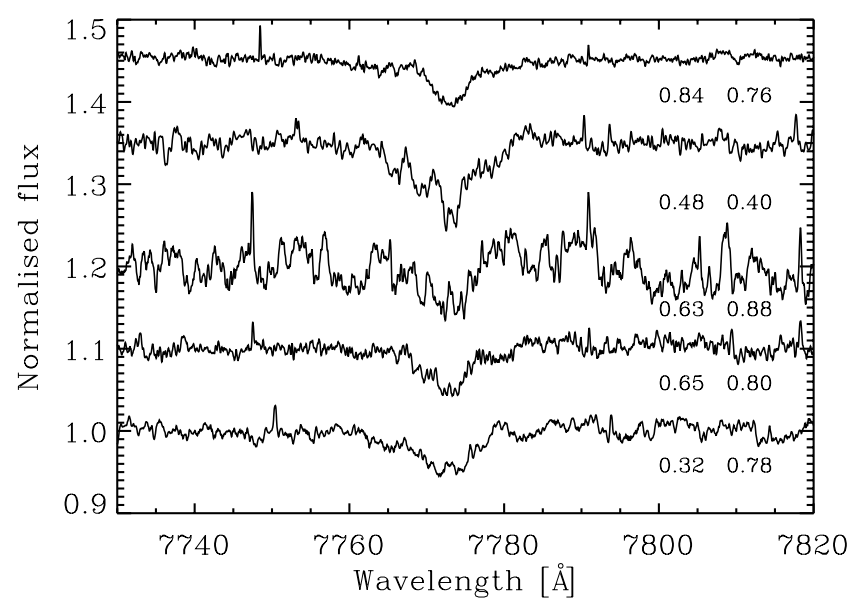

Fig. 6. Donor-subtracted spectra of DQ Vel in the OI $7773 \AA$ region. Labels indicate orbital (left) and long-term (right) phases.

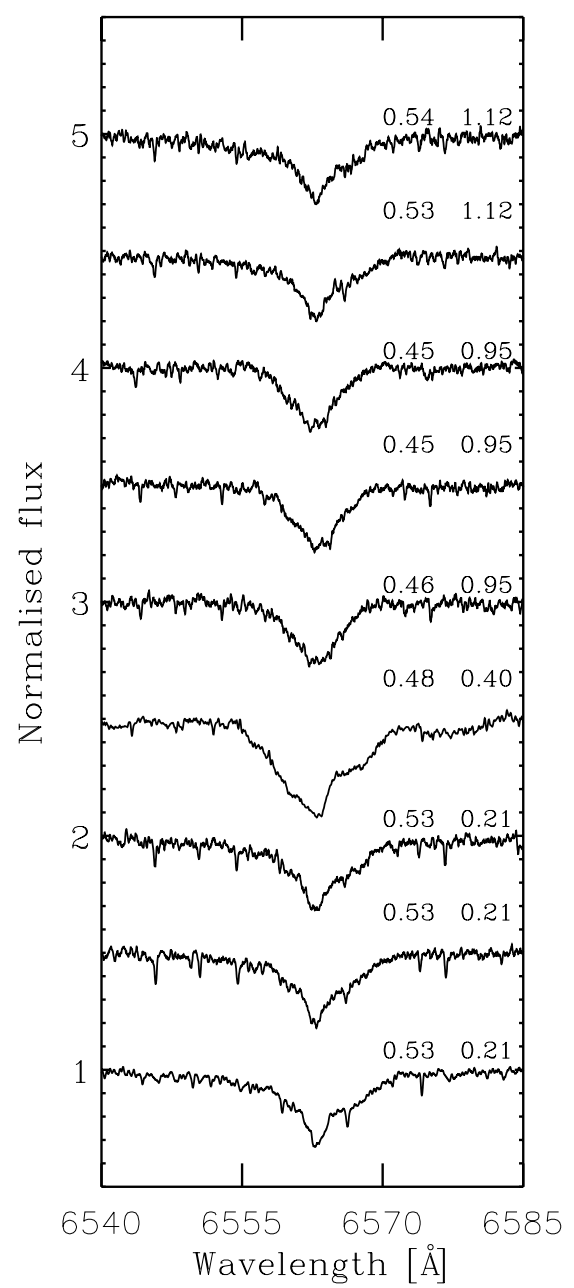

Fig. 7. Sequence of $\mathrm{H} \alpha$ composite spectra for DQ Vel around the secondary eclipse.

of the line profile are observed. However, the weakness of these emission features compared to the continuum flux, inhibit any type of quantitative analysis of the circumstellar/binary material. Intensities or EW analysis of any extra absorption and/or emission feature in the system are not possible because of blending effects with photospheric absorption. One way to analyse these emission/absorption features is by subtracting the stellar 


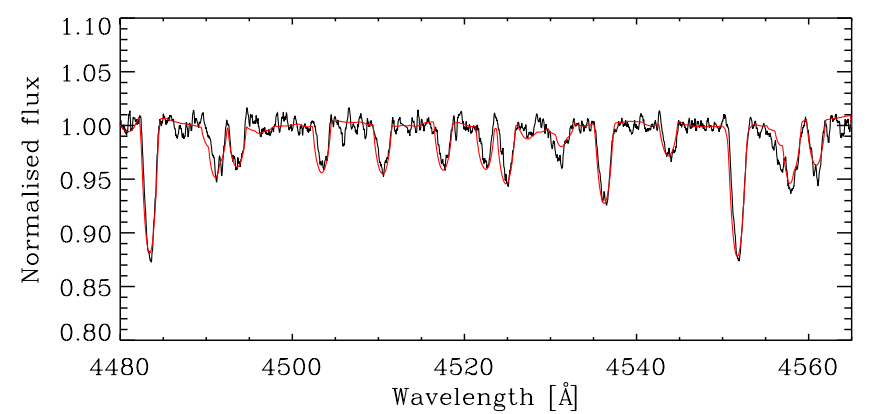

Fig. 8. Comparision between the observed (black line) and the synthetic (red line) composite spectra for DQ Vel in the blue spectral region.

photospheric absorption profiles from the observed spectra to produce the so-called difference profiles (Richards 1993).

If the helium lines observed in DQ Vel spectra are representative of the gainer motion, then we can use the gainer stellar parameters obtained from the light curve fit to generate a gainer synthetic spectrum. This can be added to the donor template to create a synthetic composite spectra for DQ Vel. From the grid of synthetic spectra (see Sect. 3.1) we select a gainer spectrum with $T_{\mathrm{d}}=18500 \mathrm{~K}, \log g_{\mathrm{d}}=4.1, v_{\mathrm{r}} \sin i=120 \mathrm{~km} \mathrm{~s}^{-1}$, and solar metallicity. At different epochs, donor and gainer synthetic spectra were corrected by RV shifts and multiplied by a light contribution factor before being summed.

In Fig. 8 is displayed a comparision between the observed and synthetic (donor+gainer) spectra in the blue spectral region. In the left-hand panel of Fig. 9 a sequence of $\mathrm{H} \alpha$ observed and synthetic composite spectra at different long-term phases are displayed. The right-hand panel shows their difference spectra. An irregular emission/absorption profile is observed at the minimum of the long-term variability $\left(\phi_{1}=0.40\right)$. At the high state $\left(\phi_{1}=0.95,1.12\right.$, and 1.21$)$ a regular single-peaked emission seems to dominate the difference spectra.

We measured the total EWs for these emission/absorption components using multiple Gaussian fits and plotted these against the long-term phase as is shown in Fig. 10. Higher EW values are found at the long-term phases $\phi_{1}=0.12$ and 0.21 and the lowest EW measurement is found at the low state $\phi_{1}=0.4$. This result supports the EW variations found in the central absorption (CA) of Balmer and helium lines at the donor-subtracted spectra (see Sect. 3.1). At higher $\mathrm{H} \alpha \mathrm{CA}$ intensities lower emission EW values. Variations on the circumstellar material emissivity support the scenario for an extra-emission appearing on the system during the long-term variability.

\subsection{Evolutionary stage}

The donor and gainer physical parameters can be used to analyse the stellar locations in the HR diagram. In Fig. 11, we display the DQ Vel components together with a set of theoretical evolutionary tracks obtained from Bressan et al. (1993) for single stars with $z=0.02$ and for different stellar masses. A first inspection shows an over-luminous donor star considering its mass of $M_{\mathrm{d}}=2.2 M_{\odot}$ and $\log g_{\mathrm{d}}=2.9$. This scenario can be explained if we consider that the donor is an evolved star that has swollen and cooled. Furthermore, the large radius found for the donor $\operatorname{star}\left(R_{\mathrm{d}}=8.4 R_{\odot}\right)$ supports this assumption. The observed light curve variations outside the eclipses together with the donor rotational projected velocity of $v_{\mathrm{d}} \sin i=69 \mathrm{~km} \mathrm{~s}^{-1}$ (Barría et al. 2013) suggest a non-spherical and synchronous rotator star that is filling its Roche lobe.
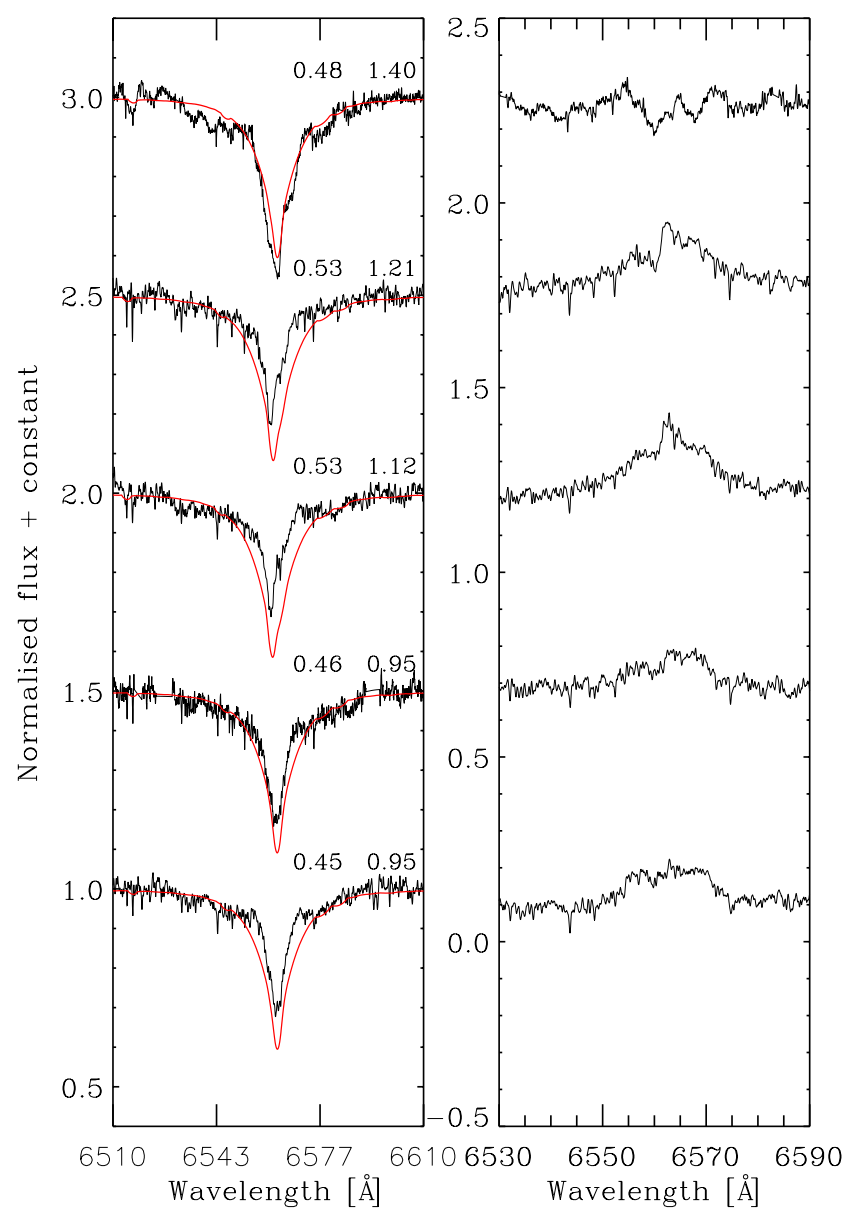

Fig. 9. Left-panel: observed and synthetic (donor+gainer) spectra of DQ Vel at different long-term phases during the secondary eclipse. The labels indicate the orbital (left) and long (right) phases. Right panel: $\mathrm{H} \alpha$ difference profiles (observed - synthetic).

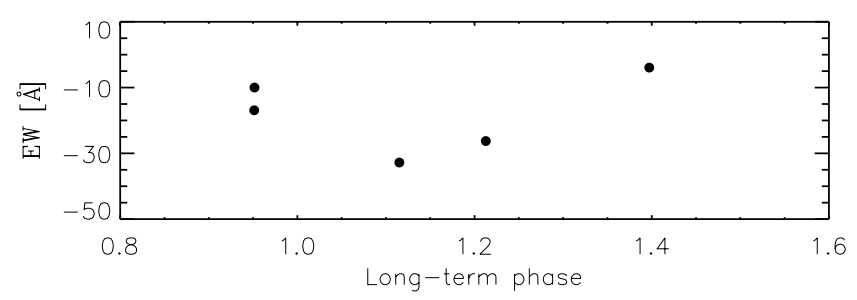

Fig. 10. Equivalent width of $\mathrm{H} \alpha$ residual spectra displayed in Fig. 9.

The gainer with a mass of $M_{\mathrm{g}}=7.3 M_{\odot}$ and $\log g_{\mathrm{g}}=4.2$ (Barría et al. 2013) appears as an under-luminous and cooler star. We can explain the lower luminosity and temperature values as a gainer star that is surrounded by circumstellar material. Underluminous gainers have also been found in others DPVs such as AU Mon (Djurasevic et al. 2010) and V393 Sco (Mennickent et al. 2012a).

We compare the physical parameters of DQ Vel with the absolute parameters of 61 semidetached Algol-type binaries analysed by Ibanoğlu et al. (2006). Two binary systems in the sample (BM Ori and RY Per) show similar properties to those of DQ Vel such as the orbital period, mass ratio, radii and temperatures. However, the stellar components of DQ Vel are more massive than the donor and gainer star of both Algol systems. A comparision of the absolute parameters of DQ Vel with those of the total semidetached systems analysed by the 
D. Barría et al.: Exploring the long-term variability and the evolutionary stage of DQ Velorum

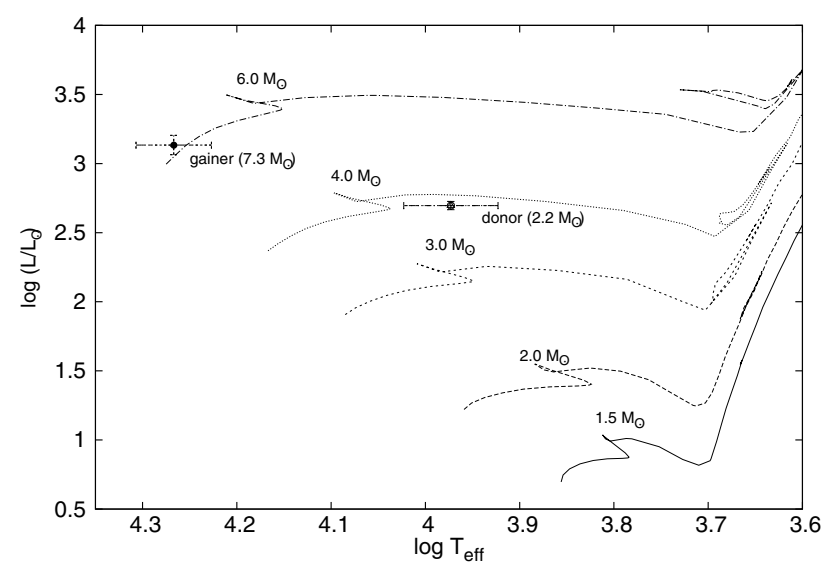

Fig. 11. Theoretical evolutionary tracks for single stars together with the DQ Vel donor and gainer locations in the HR diagram. Labels indicate different stellar masses.

authors, shows that DQ Vel follow the general tendency of classical semidetached Algol-type with an under-luminous gainer located in the main sequence band and an over-luminous donor evolved off from the main sequence with respect to single ZAMS and TAMS main sequence stars. However, after compararing DQ Vel with the total semidetached Algol systems in the planes $\log M-\log R, \log M-\log T, \log R-\log T, \log M-\log L$, and $\log P_{\mathrm{o}}-\log L$ (see Figs. 3 and 4 in Ibanoğlu et al. 2006), we found that the DQ Vel components are located at the upper limit regions in all diagrams with more luminous, more massive, and hotter stars than the average values given for the Algols sample.

The evolutionary history of DQ Vel can be predicted from a comparision of the current stellar parameters with binary evolutionary models. A catalogue of 561 evolutionary tracks for binaries which includes mass loss at some stage of the binary history were computed by Van Rensbergen et al. (2011) and are available at the Centre de Données Stellaires (CDS). This set of evolutionary tracks were calculated considering a conservative binary evolution (no mass loss from the system) and a liberal scenario where mass and angular momentum are lost during episodes of rapid mass transfer after the onset of RLOF. In the former, the gainer star rotates synchronously $\left(f_{\mathrm{g}}=1.0\right)$ and, in the latter, the gainer has spin up and its rotation is nonsynchronous $\left(f_{\mathrm{g}}=0.1\right)$. For the liberal case, each model was computed under a weak and a strong tidal interaction regime.

In Barría et al. (2013) the stellar parameters of DQ Vel were obtained by modelling the $V$-band light curve under two scenarios: a gainer under a synchronous rotation regime $\left(f_{\mathrm{g}}=1.0\right)$ and a gainer star in a critical rotation scheme where $f_{\mathrm{g}}=12.8$. At that time, no significant differences (within the errors) where found for the two fitting solutions. Here, through a multi-parametric fit, we compare each theoretical evolutionary model with the stellar and orbital parameters obtained for a synchronous and a critical rotator gainer. The observed parameters such as mass, radius, temperature, luminosity, and orbital period are compared with the theoretical values, while the mass loss rate, Roche lobe radius, chemical composition, fraction of accreted mass, and age, are free parameters. To discriminate between the different solutions to the fit, we calculate the minimum value for the quantity $\chi_{i, j}^{2}$ which is defined by

$\chi_{i, j}^{2}=\frac{1}{N} \Sigma_{k} w_{k}\left[\left(S_{i, j, k}-O_{k}\right) / O_{k}\right]^{2}$,
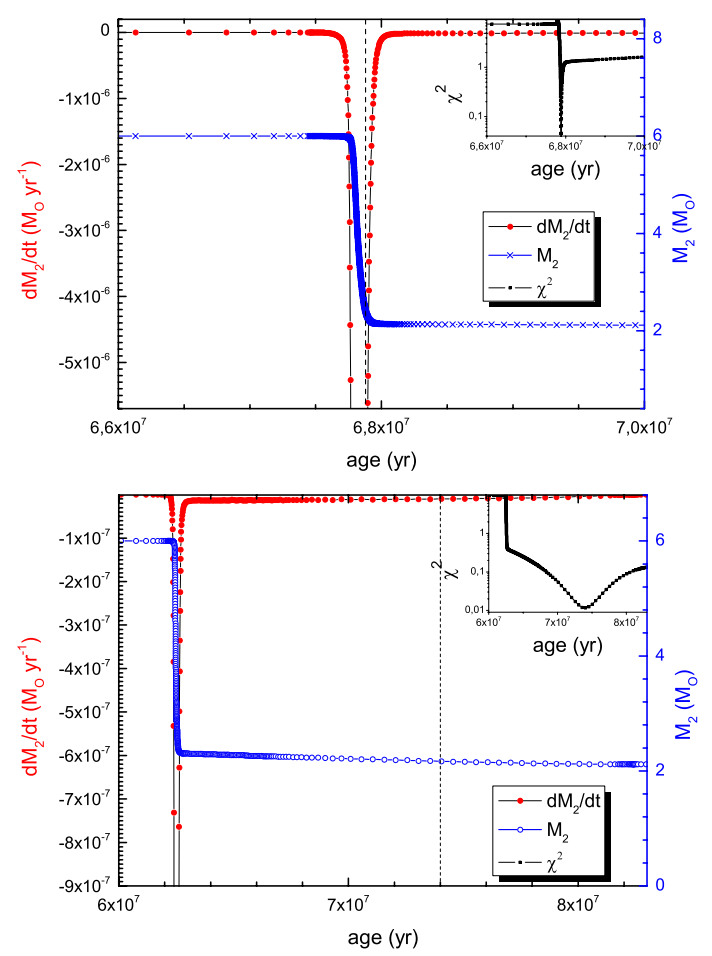

Fig. 12. Best-fit results for the evolution of DQ Vel with a gainer star under a synchronous rotation regime (upper panel) and with a gainer star under a critical rotation regime (lower panel). The vertical dashed line represents the current stage of DQ Vel.

where $O_{k}$ is the observed kth stellar parameter, $S_{i, j, k}$ represents the ith synthetic model at the time $t_{j}, N$ is a normalisation factor, and $w_{k}$ a statistical weight related to the error associated with the observed parameter $O_{k}$ (Mennickent et al. 2012a). The minimum value for $\chi_{i, j}^{2}$ corresponds to the best evolutionary model for DQ Vel. In Fig. 12, we show the best match (lower values for $\chi_{i, j}^{2}$ ) for the evolution of DQ Vel with a gainer star under a synchronous and a critical rotation scenario. The crosses conected by a line represent the time evolution of the donor mass and the circles joined by a line are the mass transfer rate. The vertical dashed line represents the current stage of DQ Vel. In Table 3, we list the best theoretical stellar and orbital parameters obtained for each case.

\subsubsection{Gainer under a synchronous rotation regime}

For a gainer star rotating synchronously with the orbital motion, the best solution is obtained for a binary under a conservative evolution regime. In this case, the present state of DQ Vel is just inside a rapid mass transfer episode with a high mass transfer rate value of $\dot{M}_{\mathrm{d}}=-8.6 \times 10^{-6}\left[M_{\odot} / \mathrm{yr}\right]$ (upper panel at Fig. 12). Such a high mass transfer rate, under a conservative scheme, would imply a significant change in the orbital period. This change rate will be given by Hilditch (2001)

$\frac{\dot{P}}{P}=\frac{3 \dot{M}_{\mathrm{d}}\left(M_{\mathrm{d}}-M_{\mathrm{g}}\right)}{M_{\mathrm{d}} M_{\mathrm{g}}}$.

Using the stellar masses and the orbital period obtained for a synchronous rotator gainer and the calculated value of the mass transfer rate, we find a change in the orbital period of $4 \mathrm{~s}$ per year. Considering a current accuracy of $10^{-6}$ on the determined orbital period, such a change should be detected with the current astronomical instruments. Furthermore, we can estimate 
Table 3. The two best theoretical solutions for the stellar and orbital parameters of DQ Vel.

\begin{tabular}{lcr}
\hline \hline Gainer regime & Synchronous & Critical \\
\hline Parameter & Value & Value \\
\hline$\chi_{i, j}^{2}$ & 0.0172 & 0.0116 \\
Age [yr] & $6.78 \times 10^{7}$ & $7.40 \times 10^{7}$ \\
Period [days] & 6.120 & 6.057 \\
$M_{\mathrm{d}}\left[M_{\odot}\right]$ & 2.351 & 2.168 \\
$\dot{M}_{\mathrm{d}}\left[M_{\odot} / \mathrm{yr}\right]$ & $-8.6 \times 10^{-6}$ & $-9.8 \times 10^{-9}$ \\
$\log T_{\mathrm{d}}[\mathrm{K}]$ & 3.89 & 3.94 \\
$\log L_{\mathrm{d}}\left[L_{\odot}\right]$ & 2.40 & 2.54 \\
$R_{\mathrm{d}}\left[R_{\odot}\right]$ & 8.69 & 8.22 \\
$X_{\mathrm{cd}}$ & 0.06 & 0.07 \\
$Y_{\mathrm{cd}}$ & 0.91 & 0.90 \\
$\log T_{\mathrm{g}}[\mathrm{K}]$ & 4.32 & 4.31 \\
$\log L_{\mathrm{g}}\left[L_{\odot}\right]$ & 3.38 & 3.46 \\
$M_{\mathrm{g}}\left[M_{\odot}\right]$ & 7.25 & 7.43 \\
$R_{\mathrm{g}}\left[R_{\odot}\right]$ & 3.69 & 4.39 \\
$X_{\mathrm{cg}}$ & 0.64 & 0.52 \\
$Y_{\mathrm{cg}}$ & 0.34 & 0.45 \\
\hline
\end{tabular}

Notes. On the left side we list the parameters with a gainer star in synchronous rotation regime and on the right side are listed the parameters for a critical rotator gainer star.

from the model that DQ Vel has remained inside this high mass transfer episode for the last $\sim 1.5 \times 10^{5}$ years. If we consider this mass transfer rate as a lower limit, we should expect at least a four-minute change in the binary orbital period during the last 60 years. A comparision between the orbital period of 6.08337(13) d found by van Houten (1950) with the current orbital period value, argues against this scenario.

\subsubsection{Gainer under a critical rotation regime}

The best evolutionary history of DQ Vel with the gainer under critical rotation (lower $\chi_{i, j}^{2}$ ) is achieved for a liberal scenario under a weak tidal interaction regime (lower panel in Fig. 12). The theoretical stellar parameters obtained in this case are listed in the right column in Table 3 . The better match between the observed and theoretical stellar properties is displayed in the lower panel in Fig. 13. Here, theoretical evolutionary tracks and physical parameters (black squares) for the donor and gainer are plotted together with the observed stellar parameters (black dots with error bars). As a comparision, in the upper panel in Fig. 13 we show the theoretical evolutionary tracks and stellar parameters for the gainer and donor under a conservative evolution scenario. Higher discrepancies between the synthetic and observed parameters are found under this regime.

As can be seen in the lower panel in Fig. 13 the observed gainer appears under-luminous and under-heated with respect to the synthetic star. One possible explanation for this might be that a fraction of the mass transferred from the donor has not been accreted by a rapidly rotating gainer and remains in the form of a gaseous envelope or of an accretion disc around the more massive star.

To estimate the projected rotational velocity of the gainer $\left(v_{\mathrm{g}} \sin i\right)$ we compare the helium profiles of the separated spectra with a grid of synthetic spectra at different rotational velocities. The best match is obtained for a synthetic spectrum with $v_{\mathrm{g}} \sin i=120 \pm 25 \mathrm{~km} \mathrm{~s}^{-1}$. This value is around four times higher than the synchronous rotational velocity in agreement with our previous results for a gainer that is not rotating synchronously.
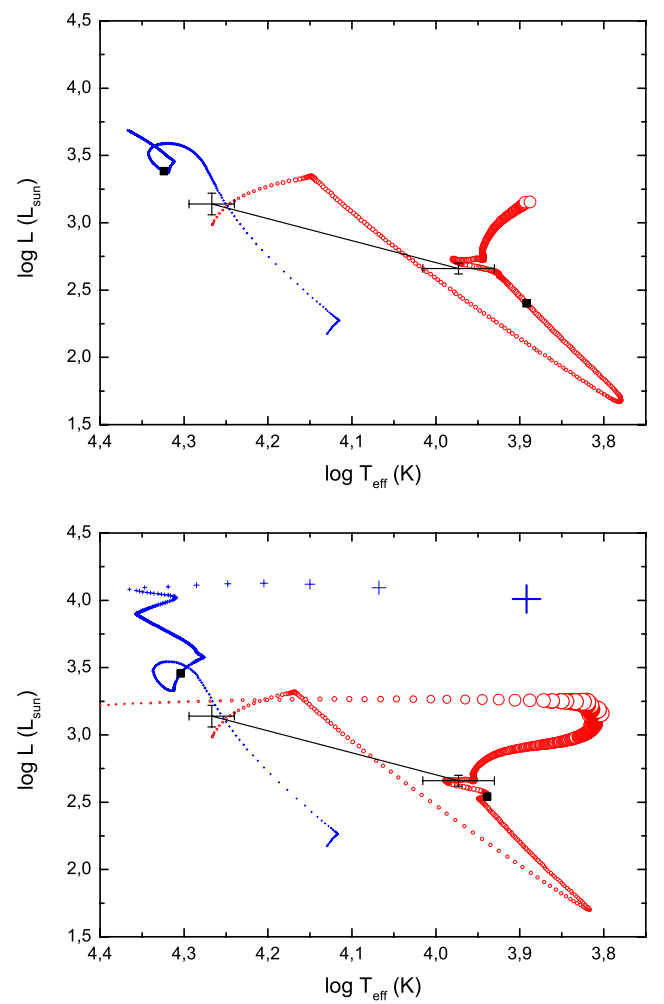

Fig. 13. Evolutionary tracks for the DQ Vel components obtained from the best evolutionary model with a synchronous rotator gainer star (top panel) and a critical rotator gainer star (lower panel). Black squares and dots with error bars represent the current theoretical and the observed parameters, respectively.

However, it is important to consider here that a possible contamination in the helium lines due to the circumstellar material may cause uncertainties in this velocity estimation.

The synchronisation time scale for DQ Vel can be estimated by Hilditch (2001)

$t_{\text {sync }} \approx 10^{4}\left(\frac{1+q}{2 q}\right)^{2} P_{\mathrm{o}}^{4}$ [years] $\approx 3.4 \times 10^{6}$ [years $]$,

where $q$ represents the system mass ratio. However, evolutionary studies suggest that DQ Vel is an older system (see Fig. 12) and thus we should expect that the stellar rotation periods have already been synchronised with the orbital period. A faster rotating gainer star can be explained then as a result of mass transfer processes in the system. The rotational parameter $F\left[\left(v_{\mathrm{g}} \sin i\right)_{\mathrm{obs}} /\left(v_{\mathrm{g}} \sin i\right)_{\text {sync }}\right] \sim 4$ for DQ Vel gainer is consistent with the $F$ values of gainer for classical Algol systems with $P_{\mathrm{o}}>5 \mathrm{~d}$ given by Soydugan et al. (2013) (see Fig. 9).

\subsection{Accretion disc}

To check the possible formation of an accretion disc, we use the mass ratio and the current orbital separation to compute the distance of closest approach (measured from the centre of the gainer) of a stream coming from the inner Lagrange point $L_{1}$. From Lubow \& Shu (1975), we obtain

$r_{\min }=0.0488 q^{-0.464} a$

$r_{\min }=(0.084 \pm 0.011) a$.

The currently observed gainer radius $R_{\mathrm{g}}=(0.135 \pm 0.05) a$ is a limiting case (within the errors) to naturally form an accretion 
disc. To investigate if an accretion disc might have been formed in the past, we explore the evolutionary history of DQ Vel at different ages to determine if the condition $R_{\mathrm{g}}<r_{\min }$ was achieved before. At present, this condition has not been fulfilled. However, the uncertainties in the $q$ and $R_{\mathrm{g}}$ values do not allow us to unambiguously reject a prior natural formation of a disc. In addition, the fact that the best light curve model is found for a semidetached configuration that includes an extended accretion disc argues against this fact. Furthermore, we cannot exclude a different mechanism to form an accretion disc around the gainer. One possibility is that during a high mass transfer rate episode, the gainer was not able to accrete all the material coming from the donor, reaching a critical rotation where a fraction of the transfered mass was accumulated close to the star. Once the mass transfer rate decreases, the gainer may slow down and the accumulated matter may spreads forming an accretion disc. The tidal interaction with the donor will continuosly break the rotational velocity of the gainer leading the material to move outwards creating an extended disc. These assumptions, together with the lower mass transfer rate $\left(-9.8 \times 10^{-9} M_{\odot} / \mathrm{yr}\right)$ found for a non-syncronous gainer, seem to be a better representation of the current observed properties of DQ Vel. As a comparision, V393 Sco is a younger system just after a high mass transfer rate episode where most likely the gainer is currently rotating critically with most of the transferred mass accumulated close to the gainer, forming a massive disc whose thickness is higher in the inner part than in the outer rim of the disc (see Table 8 in Barría et al. 2013).

\subsection{Mass transfer rate}

Ibanoğlu et al. (2012) found significant carbon deficiencies in the mass-gaining primaries of some classical Algol binaries. The authors measured the EWs of the C II $4267 \AA$ line in 18 Algol primaries and compared them to the EWs of single standard stars having the same effective temperatures. The EWs of the Algols were significantly smaller than the standard stars. This result suggests that the primary components of some Algol systems are $\mathrm{C}$ poor stars due to the mixing processes in the primary atmospheres. Since the transferred material from the evolved donor star is $\mathrm{C}$ poor, when this is mixed with the original matter of the gainer the $\mathrm{C}$ amount appears to decrease (de Greve \& Sarna 1997). Thus, Ibanoğlu et al. (2012) found a correlation between the EWs of the C II $4267 \AA$ line and the mass transfer rate. As the mass transfer rate increases, the EW of C II $4267 \AA$ decreases. Interestingly, in the Algols analysed by the authors the weakest line was found in the DPV system AU Mon (originally classified as an Algol-type star) where the EW of the C II line was only 20 per cent that of a standard star with similar effective temperature. This result suggests that AU Mon has a higher mass transfer rate value than the classical Algol systems studied by the authors.

To obtain an independent estimation of the mass transfer rate in DQ Vel, we measured the $E W / \lambda$ for the C II $4267 \AA$ line at five donor-subtracted spectra collected at orbital phase $\phi_{0}=$ 0.5 when the donor star is being eclipsed. Using the correlation $E W s / \dot{M}$ for the Algols studied by Ibanoğlu et al. (2012) we measured a mean value of the $E W / \lambda$ of $-4.7 \pm 0.3$ and thus we estimate a mass transfer rate around $\dot{M}=-5 \times 10^{-8} M_{\odot} / \mathrm{yr}$. This mass transfer rate is lower than the value predicted from the evolutionary analysis of DQ Vel with the gainer star in synchronous rotation (see Sect. 3.4.1 and Table 3), but is closer to the mass transfer rate estimated for a gainer in critical rotation $\left(-9.8 \times 10^{-9} M_{\odot} / \mathrm{yr}\right)$. Considering that the gainer star in DQ Vel is rapidly rotating $\left(120 \mathrm{~km} \mathrm{~s}^{-1}\right.$ ), but not critical (see Sect. 3.4.2), we suggest that $\dot{M} \sim 10^{-8} M_{\odot} / \mathrm{yr}$ is a better estimation of the mass transfer rate of this system.

We compare the observed EW for the C II $4267 \AA$ line with that of a synthetic star with the same effective temperature and luminosity class of the DQ Vel gainer. The observed EW of the C II $4267 \AA$ line is around 40 percent of the EW measured in the synthetic star. Comparing this result with that of AU Mon (see text above), we suggest that C in the DQ Vel gainer atmosphere is not as diluted as in the AU Mon gainer atmosphere and, thus the mass transfer rate should be higher in AU Mon than in DQ Vel.

\section{Conclusions}

We have analysed a series of optical spectra of DQ Vel during much of its long-term cycle. We have found remarkable profile variations in the donor-subtracted and in the difference spectra at similar orbital phases, suggesting that an additional source is responsible for these variabilities. The EWs of the central absorption measured in Balmer and helium lines seems to be modulated with the long cycle. During the minimum of the long-term variability we observe a strengthening in the EWs in all analysed spectral features. Moreover, during the long-term maximum all profiles appear weakened. This pattern is most remarkable at the main eclipse when the gainer star and the disc are being eclipsed by the donor star. The constancy of the orbital light curve during the long cycle suggests that this variability pattern is not related to changes in the disc properties. Furthermore, the additional source is not eclipsed and thus seems to be restricted to some region above/below the orbital plane. We suggest that the spectral variabilities observed during the long-term phase are better represented by an extra line emission produced during the maximum brightness of the system which decreases the EWs at the long-term maximum. A similar behaviour was also observed in the DPV system V393 Sco.

Discrete absorption components were found at three donorsubtracted spectra in the O I $7773 \AA$ absorption line; DACs were detected at the orbital phases $\phi_{0}=0.32,0.48$, and 0.65 together with blue enhanced absorption wings observed at the main absorption profile. Enhanced absorption wings and DACs seems to be correlated: DACs are only present when a blue or red enhanced absorption wing is observed. We suggest that DACs and enhanced absorption wings can be associated with a clumpy wind coming from the accretion disc.

The stellar parameters of DQ Vel obtained for a gainer star in synchronous and critical rotation were compared with a grid of theoretical evolutionary tracks. These tracks were computed under two different evolutionary scenarios: a conservative and a non-conservative (liberal) regime. A better representation of the current observed parameters of DQ Vel was found for a nonconservative evolutionary case, where mass has been lost for the system at some stage of the binary history. The best nonconservative evolutionary model for DQ Vel was found with the gainer star in critical rotation and the donor transfering mass at a rate of $-9.8 \times 10^{-9} M_{\odot} / y r$.

In an independent way, we measured the EW of the C II $4267 \AA$ line and estimated a higher mass transfer rate of $-5 \times 10^{-8} M_{\odot} / \mathrm{yr}$. The EW of the C II $4267 \AA$ line is around 40 per cent lower than that of a synthetic star with the same effective temperature and luminosy class of the DQ Vel gainer. After comparing the gainer helium lines in the separated 
spectra with a grid of synthetic spectra, we estimated a gainer rotational velocity of $v_{\mathrm{g}} \sin i=120 \mathrm{~km} \mathrm{~s}^{-1}$, which is around four times higher than the synchronous rotational velocity. This result however assumes that the helium lines used to estimate $v_{\mathrm{g}} \sin i$ comes from the photosphere of the gainer.

After comparing the evolutionary stages of DQ Vel and V393 Sco, we have found that DQ Vel seems to be an older system with a lower mass transfer rate. DQ Vel and V393 Sco show similar stellar parameters, but the geometrical and physical properties of their accretion discs are different. The gainer star in DQ Vel seems to be a rapidly rotating star while the V393 Sco gainer is a critical rotator. We suggest that in the older system, DQ Vel, the material transfered from the donor has had more time to spread out around the gainer and has formed an extensive accretion disc. On the other hand, the massive disc observed in V393 Sco can be explained by a younger system with a higher mass transfer rate where the critical rotator gainer might not have had enough time to accrete all the matter coming from the donor and thus to form a massive accretion disc.

Acknowledgements. We thank to Sandro Villanova, Guisella Moreno, AndréNicolas Chené, Matias Jones and Paz Bluhm for carrying out part of the observations used in this work. D.B. acknowledges support by the Chilean CONICYT Ph.D. grant. R.E.M. acknowledges support from FONDECYT grant 1110347 and project VRID 214.016.001-1.0. D.B. and R.E.M. acknowledge support from the BASAL Centro de Astrofísica y Tecnologias Afines PFB-06/2007.

\section{References}

Barría, D., Mennickent, R. E., Schmidtobreick, L., et al. 2013, A\&A, 552, 63 Bressan, A., Fagotto, F., Bertelli, G., \& Chiosi, C. 1993, A\&AS, 100, 647
Castelli, F., \& Hubrig, S. 2004, A\&A, 425, 263

Danezis, E., Nikolaidis, D., Lyratzi, V., et al. 2003, Ap\&SS, 284, 1119

de Greve, J. P., \& Sarna, M. J. 1997, ASPC, Conf. Ser., 130, 75

Djurašević, G., Latković, O., Vince, I., \& Cséki, A. 2010, MNRAS, 409, 329

Hilditch, R. W. 2001, An Introduction to Close Binary Stars (Cambridge University Press)

Hoffmeister, C. 1949 Astr. Abh. Ergänz. Astr. Nach., 12, A24

Ibanoǧlu, C., Soydugan, F., Soydugan, E., \& Dervişoğlu, A. 2006, MNRAS, 373, 435

Ibanoğlu, C., Dervişoğlu, A., Çakrl, Ö., Sipahi, E., \& Yüce, K. 2012, MNRAS, 419, 1472

Kurucz, R. 1993, CD-ROM, 18

Lubow, S. H., \& Shu, F. H. 1975, ApJ, 198, 383

Mennickent, R. E., \& Kołaczkowski, Z. 2010, ASPC, 435, 283

Mennickent, R. E., Pietrzyński, G., Díaz, M., \& Gieren, W. 2003, A\&A, 399, L47

Mennickent, R. E., Kołaczkowski, Z., Michalska, G., et al. 2008, MNRAS, 389, 1605

Mennickent, R. E., Kołaczkowski, Z., Graczyk, D., \& Ojeda, J. 2010, MNRAS, 405, 1947

Mennickent, R. E., Djurašević, G., Kołaczkowski, Z., \& Michalska, G. 2012a, MNRAS, 421, 862

Mennickent, R. E., Kolaczkowski, Z., Djurašević, G., et al. 2012b, MNRAS, 427, 607

Michalska, G., Mennickent, R. E., Kołaczkowski, Z., \& Djurašević., G. 2010, ASPC, 435, 357

Pojmanski, G. 1997, Acta Astron., 47, 467

Poleski, R., Soszyñski, I., Udalski, A., et al. 2010, Acta Astron., 60, 179

Richards, M. T. 1993, ApJS, 86, 255

Sbordone, L. 2005, Mem. Soc. Astron. It. Supp., 8, 61

Soydugan, F., Soydugan, E., Kanvermez, Ç., \& Liakos, A. 2013, MNRAS, 432, 3278

van Houten, C. J. 1950, AnLei, 20, 223

Van Rensbergen, W., De Greve, J. P., Mennekens, N., Jansen, K., \& De Loore, C. 2011, A\&A, 528, 16 